holograms (for image-synthesis, coding and interferometry), and now a new type of "reflection hologram" observable in white light.

G W Strokes

Electrical Engineering Department

The University of Michigan

Ann Arbor, Michigan

\section{Recent results in holography}

A survey of the current holographic activity in our laboratory is given. The topics covered are: theory of holographic interferometry, its application to vibration and surface deformation measurements, surface contour generation on holographic images, the use of threedimensional storage media for holographic recording, the animation of holographic images, and the attainment of high quality imagery through diffusing media.

EN Leith

Electrical Engineering Department

The University of Michigan

Ann Arbor, Michigan

\section{Lasers for length measurement}

Because of their high intensity and coherence, lasers are superior light sources for interferometric length measurements. Good wavelength stability as well as an accurate knowledge of the laser wavelength are prerequisites to provide the high precision required of such measurements.

Experiments will be described which led to the first successful measurement of a standard metcr bar with a helium-neon laser used as a light source.

K D Mielenz

National Bureau of Standards

Washington, $D C$

\section{Session II}

Moderator: Dr Harold Katz (Tecumseh Products, Ann Arbor, Michigan)

\section{The use of lasers in signal processing for radar and communications} Lasers have been used in a large variety of signal processing optical equipments. The basic ideas will be reviewed and then applied to a number of problems in radar processing and communications signal processing.

The major ideas involving the use of optics for radar will be applied to the process of generating a synthetic antenna whercby a resolution significantly finer than that of a radiated beam-width is achieved. In the application of optics to communications, it is shown that many of the coding-decoding and synchronization operations can be mechanized optically.

\section{J Cutrona}

Electrical Engineering Department

The University of Michigan and Conductron Corporation

Ann Arbor, Michigan

\section{Airborne investigations of clear-air turbulence with laser radars}

Over 1300 experiments have been performed with a laser radar mounted in a light twin-engine airplane. Ancillary equipment included an accelerometer, thermistor, data recording equipment, and a forward-looking camera for recording the local meteorology. The studies were performed in mountain waves near Denver, in the severe storm activity of Oklahoma, and the local Detroit area. A description of the equipment will be given, together with examples of the data. $P$ A Franken, J A Jenney and D M Rank

Physics Department

The University of Michigan

Ann Arbor, Michigan

Zapping paper for monitoring the temporal and spatial distribution of laser energy

A novel and simple method for monitoring the temporal and spatial distribution of pulsed laser energy by means of a multilayer coated paper is described.

J H Myer

Hughes Aircraft Company

Newport Beach

California
The application of lasers in thermophysical properties measurements The application of a ruby laser as the pulsed energy source for the flash technique of measuring the thermophysical properties of materials is discussed. The advantages and limitations of its application, as well as other related problems, are treated and the results of measurements performed are presented.

M M Nakata

Atomics International

Canoga Park, California

Charge compensator site' ln laser $\mathrm{CaWo}_{4}$

This paper is intended to give certain preliminary results of the program to investigate the role of charge compensators in laser $\mathrm{CaWo}_{4}$. Vanadium, which lies above niobium in the periodic table, is introduced as the charge compensator and micro-probe. Electron spin resonance measurements show that vanadium occupies W-and/ or Ca-sites depending upon the relative concentration of vanadium to rare-earth. These and the preliminary optical results will be discussed.

C Kikuchi and N Mahootian

Department of Nuclear Engineering

University of Michigan

Ann Arbor, Michigan

W Wiehmann

Harry Diamond Laboratories

Washington, $D C$

The effect of internal reflection in a corner cube upon the polarization of a reflected beam

Graphical and matrix methods are presented for analyzing the change in state of polarization of a light beam which undergoes successive internal reflections. The techniques are applied to the design of a laser cavity incorporating internal reflectors which are both selfaligning and highly efficient.

$P$ J Walsh and I Krause

University Associates, Union

New Jersey

\section{Session III}

Moderator: Dr A B El-Kareh (The Pennsylvania State University, University Park, Pennsylvania)

\section{Laser machining}

This paper presents the results of an experimental program to define the current status of pulsed lasers as machine tools for material removal. The laser machining systems used for the test were sufficiently flexible so that an extensive study could be made of the effects of energy, power, pulse duration, beam divergence and focusing of the beam on machining capability. The machining tests were limited to the following materials: Rene-41, Inconel 713, L-605, Titanium 6-4, Aluminium Oxide, Hastelloy X, C-2 Carbide, Stellite 19, and D-6 Steel.

W V Trammel

GM Defense Research Laboratories

Santa Barbara, California

Laser piercing of diamond dies on a production basis

This paper will describe the development of a production line facility for piercing holes in diamond wire-drawing dies with a laser. The development of a method for piercing diamonds without structural damage will be discussed. The specifications of the laser and the design characteristics of the unique closed circuit television viewing system will be covered.

R W Dyer and J P Epperson

Western Electric Company, Inc

Princeton, New Jersey

J C Grzywa

Western Electric Company, Inc

Buffalo, New York

Design of a production-worthy laser microwelder

The evolutionary steps in the design and construction of a productionworthy, safe, and economical laser microwelder are described. A novel, modular, liquid-cooled, cylindrical ruby laser head as well as 